\title{
ON THE ASYMPTOTIC INTEGRATION OF ORDINARY DIFFERENTIAL EQUATIONS
}

\section{PhILIP HARTMAN AND NELSON ONUCHIC}

1. Various methods have been employed for the asymptotic integration of ordinary differential equations, e.g., successive approximations (cf. [2]), topological arguments involving Waiewski's or similar principles (cf. [4], [5], [8]), and fixed points theorems (cf. [3]). The object of this note is illustrate the application for this purpose of a simple and general theorem which is based, on the one hand, on Massera and Schäffer's [7] use of the open mapping theorem and, on the other hand, on Tychonoff's fixed point theorem. This general theorem is essentially a corrected version of a theorem of Corduneanu [1].

Below $x, y, \cdots$ are elements of a finite dimensional Banach space $X$ of norms $\|x\|,\|y\|, \cdots, L$ denotes the space of real-valued functions $\varphi(t)$ on $J: 0 \leqq t<\infty$ with the topology of convergence in the mean $L^{1}$ on bounded intervals. $B$ denotes a Banach space of realvalued functions $\varphi(t)$ on $0 \leqq t<\infty$, norm $|\varphi|_{B}$, which is stronger than $L$ (in the sense that $B$ is contained in $L$ algebraically and convergence in $B$ implies convergence in $L$; [7]). Examples of such spaces are $L^{p}=L^{p}(0, \infty), 1 \leqq p \leqq \infty$, with norm $|\varphi|_{p}$ or the subspace $L_{0}^{\infty}$ of $L^{\infty}$ of functions $\varphi(t)$ satisfying $\varphi(t) \rightarrow 0$ as $t \rightarrow \infty$.

$L(X), L^{p}(X), B(X), \cdots$ will represent the space of measurable functions $x(t)$ from $J$ to $X$ such that $\varphi(t)=\|x(t)\|$ is in $L, L^{p}, B, \cdots$. In the case $L^{p}$ or $B$, the norm $|\varphi|_{p}$ or $|\varphi|_{B}$ will be abbreviated to $|x|_{p}$ or $|x|_{B} . C(X)$ is the space of continuous functions from $J$ to $X$ with the topology of uniform convergence on bounded intervals.

Consider a homogeneous and an inhomogeneous system of linear differential equations

$$
\begin{aligned}
& x^{\prime}=A(t) x, \\
& x^{\prime}=A(t) x+g(t),
\end{aligned}
$$

in which $g(t) \in L(X), A(t)$ is an endomorphism of $X$ for fixed $t$ and is locally integrable on $J$. If $\mathscr{D}$ is a Banach space stronger than $L(X)$, a $\mathscr{D}$-solution $x(t)$ of $(1.1)$ or $(1.2)$ is a solution $x(t) \in \mathscr{D}$. Let

Received October 31, 1962, and in revised form December 27, 1962. The research of the first author was supported by the Air Force Office of Scientific Research. It was completed while the second author was a Fellow of the John Simon Guggenheim Memorial Foundation.

The authors wish to thank J. J. Schäffer for calling their attention to the paper of Corduneanu [1] . 
$X_{0 \mathscr{D}}$ denote the set of initial points $x(0) \in X$ of $\mathscr{D}$-solutions $x(t)$ of (1.1). Then $X_{0 \mathscr{D}}$ is a subspace of $X$. Let $X_{1 \mathscr{D}}$ be any subspace of $X$ complementary to $X_{0 \mathscr{D}}$ (i.e., $X$ is a direct sum of $X_{0 \mathscr{D}}$ and $X_{1 \mathscr{D}}$ ) and $P_{0 \mathscr{D}}$ the projection of $X$ onto $X_{0 \mathscr{D}}$ annihilating $X_{1 \mathscr{D}}$.

A pair $(\mathscr{B}, \mathscr{D})$ of Banach spaces, each stronger than $L(X)$, is called admissible for (1.2) or $A(t)$ if, for every $g(t) \in \mathscr{B}$, (1.2) has at least one $\mathscr{D}$-solution $x(t)$; [7], p. 292.

The results below deal with the nonlinear system

$$
x^{\prime}=A(t) x+f(t, x) .
$$

Let $\mathscr{B}, \mathscr{D}$ be Banach spaces stronger than $L(X)$. Let $\Sigma=\Sigma_{\mathscr{D} \text {. }}$ be the closed ball $\left\{x(t): x(t) \in \mathscr{D},|x|_{\mathscr{D}} \leqq \rho\right\}$ of radius $\rho>0$ in $\mathscr{D}$. Let $S=\Sigma_{n} C(X)$ and $\bar{S}$ be the closure of $S$ in $C(X)$.

Theorem 1.1. Let $A(t)$ be locally integrable on $J$. Let $A(t)$, $f(t, x)$ satisfy (a) $(\mathscr{B}, \mathscr{D})$ is admissible for $A(t)$; (b) $x(t) \rightarrow f(t, x(t))$ is a continuous map of the subset $\bar{S}$ of the space $C(X)$ into $\mathscr{B}$; (c) there exists a constant $r>0$ such that $|f(t, x(t))|_{\mathscr{B}} \leqq r$ for $x(t) \in \bar{S}$; and (d) there exists a $\lambda(t) \in L$ such that $\|f(t, x(t))\| \leqq \lambda(t)$ for $x(t) \in \bar{S}$. Let $\xi_{0} \in X_{0 g}$. Then there exist positive constants $C_{0}$ and $K$, depending; only on $A(t), \mathscr{B}, \mathscr{D}, X_{1 \mathscr{D}}$ (but not on $f$ or $\xi_{0}$ ) such that if

$$
C_{0}\left\|\xi_{0}\right\|+K r \leqq \rho,
$$

then (1.3) has at least one solution $x(t) \in S$ satisfying

$$
P_{0 \mathscr{D}} x(0)=\xi_{0} .
$$

It can be remarked that if $S \neq \bar{S}$, so that $S$ is not closed in $C(X)$, then a convenient sufficient condition for (b), (c), (d) is the following: Let $\mathscr{D}$ be a subspace of a Banach space $\mathscr{E}$, stronger than $L(X)$, such that $S_{\mathscr{E}}=C(X) \cap \Sigma_{\mathscr{E}}$ is closed in $C(X)$. Then since $\bar{S} \subset S_{\mathscr{E}}$, conditions (b), (c), (d) hold if they hold when $\bar{S}$ is replaced by $S_{\mathscr{C}}$.

Theorem 1.1 is a variant of Corduneau's Theorem II in [1]. Corduneanu's theorem does not involve the space $C(X)$ and in place of (b), has the assumptions:

(b') $x(t) \rightarrow f(t, x(t))$ is a continuous map from the subset $\Sigma$ of $\mathscr{D}$ to $\mathscr{B}$;

(e') $\quad \Sigma$ is closed in $L(X)$.

However, his proof is incomplete under his assumptions and seems to use the following condition in place of $\left(b^{\prime}\right)$ :

$\left(b^{\prime \prime}\right) x(t) f(t, x(t))$ is a continuous map from the subset $\Sigma$ of $L(X)$ to $\mathscr{B}$.

It will be clear below that, in applications, it is more convenient 
to deal with the topology of $C(X)$ rather than $L(X)$.

It can be mentioned that another result (Theorem I) of Corduneanu gives, not only existence but, also uniqueness for $x(t)$ under conditions including $|f(t, x(t))-f(t, y(t))|_{\mathscr{D}} \leqq \theta|x(t)-y(t)|_{\mathscr{D}}$ for some constant $\theta$. See $\S 6$ below.

For theorems related to Theorem 1.1 with $\mathscr{D}=L^{\infty}(X)$, see [6].

The proof of Theorem 1.1 which follows closely that of Corduneanu will be given for the sake of completeness.

Proof of Theorem 1.1. The essential part of the proof is the following result of Massera and Schäffer, [7], p. 295:

LEMma 1.1. Let $A(t)$ be as above and $(\mathscr{B}, \mathscr{D})$ admissible for (1.2). Let $\xi_{0} \in X_{0 \mathscr{D}}$. Then if $g(t) \in \mathscr{B},(1.2)$ has a unique $\mathscr{D}$-solution $x(t)$ satisfying (1.5). Furthermore, there exist constant $C_{0}$ and $K$, depending only on $A(t), \mathscr{B}, \mathscr{D}$ and $X_{1 \mathscr{D}}$, such that

$$
|x|_{\mathscr{D}} \leqq C_{0}\left\|\xi_{0}\right\|+K|g|_{\mathscr{O}} \cdot
$$

Thus, if $y(t) \in \bar{S}$ in Theorem 1.1, then

$$
x^{\prime}=A(t) x+f(t, y(t))
$$

has a unique $\mathscr{D}$-solution $x(t)$ satisfying (1.5) and (1.6), where $g(t)=$ $f(t, y(t))$. Define an operator $T_{0}: \quad \bar{S} \rightarrow \mathscr{P}$ by putting $x(t)=T_{0}[y(t)]$. Thus, by (c), the condition (1.4) implies that $T_{0}$ map $\bar{S}$ into $S$.

Let $y_{j}(t) \in \bar{S}, g_{j}(t)=f\left(t, y_{j}(t)\right), x_{j}(t)=T_{0}\left[y_{j}(t)\right]$ for $j=1,2$. Then $x_{1}(t)-x_{2}(t)$ is a $\mathscr{D}$-solution of (1.2) with $g=g_{1}-g_{2}$ and $P_{0 \mathscr{D}}\left(x_{1}(0)-\right.$ $\left.x_{2}(0)\right)=0$. Hence

$$
\left|x_{1}-x_{2}\right|_{\mathscr{D}} \leqq K\left|g_{1}-g_{2}\right|_{\mathscr{B}} \text {. }
$$

Since the map from $\bar{S} \subset C(X)$ to $\mathscr{B}$ given by $y(t) \rightarrow g(t)=f(t, y(t))$ is continuous. It follows that $T_{0}: \quad \bar{S} \rightarrow S$ is a continuous map from $\bar{S} \subset C(X)$ to $S \subset \mathscr{D}$.

It will be verified that $T_{0}: \bar{S} \rightarrow S$ is a continuous map from $\bar{S} \subset C(X)$ to $S \subset C(X)$. This will depend on a standard inequality for solutions of (1.2),

$$
\|x(t)\| \leqq\left\{T^{-1} \int_{0}^{T}\|x(s)\| d s+\int_{0}^{T}\|g(s)\| d s\right\} \exp \int_{0}^{T}\|A(s)\| d s
$$

for $0 \leqq t \leqq T$, cf. [7], p. 288. It will also depend on the fact that $\mathscr{D}$ is stronger than $L(X)$, so that there exists a constant $\alpha_{\mathscr{D}}(T)$ such that

$$
\int_{0}^{T}\|x(t)\| d t \leqq \alpha_{\mathscr{D}}(T)|x|_{\mathscr{D}} \quad \text { for all } \quad x \in \mathscr{D}
$$


and that $\mathscr{B}$ is stronger than $L(X)$, so that a corresponding inequality holds for elements $g \in \mathscr{B}$ with a constant $\alpha_{\mathscr{B}}(T)$. Thus, in the notation of (1.8), for $0 \leqq t \leqq T$,

$$
\begin{aligned}
& \left\|x_{1}(t)-x_{2}(t)\right\| \\
& \quad \leqq\left\{T^{-1} \alpha_{\mathscr{D}}(T) K+\alpha_{\mathscr{D}}(T)\right\}\left|g_{1}-g_{2}\right|_{\mathscr{P}} \exp \int_{0}^{T}\|A(s)\| d s .
\end{aligned}
$$

Hence $\left|g_{1}-g_{2}\right|_{\mathscr{B}} \rightarrow 0$ implies $x_{1}(x)-x_{2}(t) \rightarrow 0$ in $C(X)$. This proves that $T_{0}: \quad \bar{S} \rightarrow S$, where $S \subset \bar{S} \subset C(X)$, is continuous.

It will now be verified that the image $T_{0} \bar{S}$ of $\bar{S}$ under $T_{0}$ has a compact closure in $C(X)$. It follows from (1.9) that if $x(t) \in T_{0} \bar{S}$, then $\|x(t)\| \leqq c(T)$ for $0 \leqq t \leqq T$, where $c(T)=\left\{T^{-1} \alpha_{\mathscr{D}}(T) \rho+\alpha_{\mathscr{B}}(T) r\right\}$ $\exp \int_{0}^{T}\|A(s)\| d s$. Thus the functions $x(t) \in T_{0} \bar{S}$ are uniformly bounded on every interval $[0, T]$. From (1.7) and condition $(d), x(t) \in T_{0} \bar{S}$ implies that

$$
\|x(t)-x(s)\| \leqq c(T) \int_{s}^{t}\|A(u)\| d u+\int_{s}^{t} \lambda(u) d u \quad \text { for } \quad 0 \leqq s \leqq t \leqq T .
$$

Thus the set of functions $x(t) \in T_{0} \bar{S}$ are equi-continuous on every interval $[0, T]$. It follows from Arzela's theorem that $T_{0} \bar{S}$ has a. compact closure in $C(X)$.

By Tychonoff's theorem, the continuous map $T_{0}: \bar{S} \rightarrow S$, where $S \subset \bar{S}$ and $\bar{S}$ is closed convex subset of $C(X)$, has a fixed point $x(t) \in T_{0} \bar{S} \subset S$. This proves Theorem 1.1.

2. In applications, condition (a) is one of the most difficult to check. For the case $(\mathscr{B}, \mathscr{D})=\left(L^{1}(X), L^{\infty}(X)\right)$, Massera and Schäffer [7] have given necessary and sufficient conditions for (a) in terms of the solutions of the homogeneous equation (1.1). (These condition will not be used below but will be stated here because of their relevancy for this paper.) First, $\left(L^{1}(X), L^{\infty}(X)\right)$ is admissible if and only if $\left(L^{1}(X), L_{0}^{\infty}(X)\right)$ is admissible; [7], p. 331. For $\mathscr{D}=L^{\infty}(X)$ or $\mathscr{D}=$ $L_{0}^{\infty}(X)$, let $X_{0 \mathscr{D}}, X_{1 \mathscr{D}}, P_{0 \mathscr{D}}$ have the meaning above and $P_{1 \mathscr{D}}=1-P_{0 \mathscr{D}}$ and let $U(t)$ be the fundamental solution of (1.1) satisfying $U(0)=I$. Then $\left(L^{1}(X), L^{\infty}(X)\right)$ and/or $\left(L^{1}(X), L_{0}^{\infty}(X)\right)$ is admissible if and only if ([7], pp. 331, 334) there exists a constant $N_{0}$ such that

$$
\begin{aligned}
& \left\|U(t) P_{0 \mathscr{D}} U^{-1}(s)\right\| \leqq N_{0} \text { for } t \geqq s \geqq 0, \\
& \left\|U(t) P_{1 \mathscr{D}} U^{-1}(s)\right\| \leqq N_{0} \text { for } s \geqq t \geqq 0 .
\end{aligned}
$$

Conditions (b), (c), (d) are comparatively easy to check under suitable conditions on $\mathscr{B}, \mathscr{D}$ and $f(t, x)$, which will now be described.

Let $B$ be a Banach space of real-valued functions on $J$ satisfying: 
(i) $B$ is stronger than $L$;

(ii) if $\varphi(t) \in B, \psi(t)$ is measurable and $|\psi(t)| \leqq|\varphi(t)|$, then $\psi(t) \in B$ and $|\psi|_{B} \leqq|\varphi|_{B}$

(iii) if $h_{0 T}(t)$ is the characteristic function of the interval $0 \leqq t \leqq T$, then $h_{0 T} \in B$;

(iv) $B$ is lean at $\infty$ ([9], p. 362), i.e., if $\varphi(t) \in B$, then $h_{0 r}(t) \varphi(t) \in B$ by (ii) and $h_{0 T} \varphi \rightarrow \varphi$ in $B$ as $T \rightarrow \infty$.

For example the spaces, $B=L^{p}, 1 \leqq p<\infty$, and $B=L_{0}^{\infty}$ (but not $B=L^{\infty}$ ) satisfy (i) - (iv). Another example of a space $B$ satisfying (i) - (iv) can be obtained as follows: Let $\psi(t)>0$ be a measurable function on $J$ such that $\psi(t)$ and $1 / \psi(t)$ are locally bounded. Let $L_{\psi_{0}}^{\infty}$ denote the Banach space of functions $\varphi(t)$ such that $\varphi(t) / \psi(\mathrm{t}) \in L_{0}^{\infty}$ with $|\varphi|_{B}=\left.|\varphi| \psi\right|_{\infty}$. This space satisfies (i)-(iv). If the function $\lambda(t)$ satisfies

$$
0 \leqq \lambda(t) \leqq \psi(t) \text { and } \lambda(t) / \psi(t) \rightarrow 0 \text { as } t \rightarrow \infty,
$$

then $\lambda(t) \in L_{\psi 0}^{\infty}$.

Condition (H) on $f$. Let $f(t, x)$ be a function on the product. space of $J: 0<t<\infty$ and the ball $\|x\| \leqq \rho$ in $X$ and let $f(t, x)$ have values in a subspace $Y$ of $X$. Let $f(t, x)$ be a measurable function of $t$ for fixed $x$ and a continuous function of $x$ for fixed $t$, where the continuity in $x$ is uniform for $t$ on bounded intervals. Let $\lambda(t)$ be a. measurable function and let

$$
\|f(t, x)\| \leqq \lambda(t) \text { for } t \geqq 0,\|x\| \leqq \rho .
$$

The point of assuming that $f(t, x)$ has values in a subspace $Y$ of $X$ is for applications to higher order equations where $f(t, x)$ might be a vector of the form $(0, \cdots, 0, h(t, x))$ with values in a subspace $Y$ of $X$; cf. $\S 3$.

LEMMA 2.1. If $\mathscr{B}$ is a Banach space of the type $\mathscr{B}=B(Y)$, where $B$ satisfies (i)-(iv), if $\mathscr{D}=L^{\infty}(X)$, and if $f(t, x)$ satisfies conditon $(\mathrm{H})$ with $\lambda(t) \in B$, then the assumtions (b), (c), (d) of Theorem. 1.1 hold with $r=|\lambda|_{B}$ in (c).

This is clear. Hence Theorem 1.1 has the following consequence.

CoRollary 2.1. Let $A(t)$ be locally integrable on $J$ and let $(\mathscr{B}, \mathscr{D})$ be admissible for (1.2), where $\mathscr{B}=B(Y)$ and $B$ satisfies (i) - (iv) above and $\mathscr{D}=L^{\infty}(X)$ [or $\left.\mathscr{D}=L_{0}^{\infty}(X)\right]$. Let $f(t, x)$ satisfy assumption $(\mathrm{H})$ where $\lambda(t) \in B$. Let $\xi_{0} \in X_{0 \mathscr{D}}$ and $r=|\lambda(t)|_{B}$. Then if (1.4) holds, (1.3) has a solution $x(t)$ satisfying (1.5) and $\|x(t)\| \leqq$ 
$\rho[$ and $x(t) \rightarrow 0$ as $t \rightarrow \infty]$.

For the purposes of asymptotic integration or the study of asymptotic equivalence, the following particular cases of Corollary 2.1 are of interest.

THEOREM 2.1. Let $A(t)$ be locally integrable on $J$. Let $f(t, x)$ satisfy assumption $(\mathrm{H})$. Assume either (i) that $\lambda(t) \in L^{1}$ and $\left(L^{1}(Y), \mathscr{D}\right)$, where $\mathscr{D}=L^{\infty}(X)$ [or $\mathscr{D}=L_{0}^{\infty}(X)$ ], is admissible for $A(t)$ or (ii) that there exists a locally bounded measurable function $\psi(t)>0$ on $J$ satisfying (2.2), such $1 / \psi(t)$ is locally bounded, and such that for every $g(t) \in L(Y)$ for which

$$
g(t) / \psi(t) \rightarrow 0 \text { as } t \rightarrow \infty,
$$

(1.2) has a $\mathscr{D}$-solution $x(t)$. Let $\xi_{0} \in X_{0 \mathscr{D}}$. Then there exist positive constants $C_{0}$ and $K$ with the property that if $\left\|\xi_{0}\right\|$ is so small and $T$ so large that,

$$
C_{0}\left\|\xi_{0}\right\|+K \int_{T}^{\infty} \lambda(t) d t \leqq \rho
$$

or

$$
C_{0}\left\|\xi_{0}\right\|+K \lambda(t) / \psi(t) \leqq \rho \text { for } t \geqq T,
$$

according as (i) or (ii) is assumed, then the differential equation (1.3) has a solution $x(t)$ for $t \geqq T$ satisfying $\|x(t)\| \leqq \rho[$ and $x(t) \| \rightarrow$ 0 as $t \rightarrow \infty$ ] and

$$
P_{0 \mathscr{D}} U^{-1}(T) x(T)=\xi_{0} \text {. }
$$

It is clear that if condition (i) holds, then (ii) holds, but it is convenient to isolate condition (i). Theorem 2.1 follows by applying Corollary 2.1 to the situation where $f(t, x), \lambda(t)$ is replaced by $h_{T}(t) f(t, x), h_{T}(t) \lambda(t)$, respectively, where $h_{T}(t)$ is the characteristic function of the half-line $t \geqq T$.

Conditions of smallness on $\left\|\xi_{0}\right\|$ in Theorem 2.1 can be eliminated by requiring that condition $(H)$ hold for every fixed $\rho>0$ where, however, $\lambda(t)$ can be allowed to depend on $\rho$.

3. Before giving more complicated applications of Theorem 2.1, it might be well to illustrate its use in a simple case. Consider a scalar second order equation

$$
u^{\prime \prime}=h\left(t, u, u^{\prime}\right),
$$

where $h(t, u, v)$ is continuous for $t \geqq 0$ and arbitrary $(u, v)$. Let $a, b$ 
be constants and consider the question whether (3.1) has a solution, for large $t$, such that

$$
u(t)-a t-b \rightarrow 0 \text { and } u^{\prime}(t)-a \rightarrow 0 \text { as } t \rightarrow \infty .
$$

Introduce the change of variables $u \rightarrow w$,

$$
u=a t+b+w
$$

so that (3.1) becomes

$$
w^{\prime \prime}=h\left(t, a t+b+w, a+w^{\prime}\right)
$$

and (3.2) becomes $w, w^{\prime} \rightarrow 0$ as $t \rightarrow \infty$. It follows from Theorem 2.1 that (3.1) has a solution $u(t)$, for large $t$, satisfying (3.2) if there exists a $\rho>0$ and a function $\lambda(t)$ such that

$$
\begin{gathered}
|h(t, a t+b+u, a+v)| \leqq \lambda(t) \text { for }|u|,|v| \leqq \rho, \\
\int^{\infty} t \lambda(t) d t<\infty ;
\end{gathered}
$$

cf. [3].

Generalizations to an $n$th order equation $u^{(n)}=h\left(t, u, u^{\prime}, \cdots, u^{(n-1)}\right)$, are obvious.

4. Very often the question of an asymptotic integration of (1.3) can be put into the following form: Let $R(t)$ be a given family of non-singular endomorphisms of $X$ which are absolutely continuous with respect to $t$ and let $c \in X$ be a constant vector. Does (1.3) have a solution $x(t)$, for large $t$, of the form

$$
x=R(t) y
$$

where $y=y(t) \rightarrow c$ as $t \rightarrow \infty$ ? The differential equation for $y(t)$ is

$$
y^{\prime}=R^{-1}(t)\left[A(t) R(t)-R^{\prime}(t)\right] y+R^{-1}(t) f(t, R(t) y) .
$$

The change of variables

$$
z=y-c
$$

transforms (4.2) into

$$
z^{\prime}=R^{-1}(t)\left[A(t) R(t)-R^{\prime}(t)\right] z+h(t, z, c),
$$

where

$$
h(t, z, c)=R^{-1}\left[A R-R^{\prime}\right] c+R^{-1} f(t, R z+R c) .
$$

The problem now becomes: Does (4.4) have a solution $z(t)$ for large 
$t$ such that $z(t) \rightarrow 0$ as $t \rightarrow \infty$ ? Clearly, Theorem 2.1 is adapted to answer such questions.

It must be pointed out that if the answer is in the affirmative, then the assertion "(4.1) and $y-c=o(1)$ as $t \rightarrow \infty$ " need not be very informative unless estimates for $y-c=o(1)$ are obtained. For example, if $R(t)$ is the $2 \times 2$ matrix $R(t)=\left(r_{j k}\right)$, where $r_{k 1}=(-1)^{k} e^{-t}$ and $r_{k 2}=e^{t}$ for $k=1,2$, and $c=(1,0)$, then one can only deduce $x(t)=o\left(e^{t}\right)$, but not an asymptotic formula of the type $x(t)=(-1+$ $o(1), 1+o(1)) e^{-t}$.

As an illustration of this procedure, there will now be indicated a derivation of an analogue of a theorem of Hartman and Wintner [5]. This theorem, in the linear case, is a generalization of a result of Dunkel [2].

The theorem will deal with a nonlinear system

$$
x^{\prime}=C x+f(t, x) \text {. }
$$

A fixed coordinate system is assumed for $X$, so that $x=\left(x^{1}, \cdots, x^{n}\right)$, where $n=\operatorname{dim} X, C$ is a constant matrix, $f(t, x)$ is continuous on $J \times X$. (Actually, it will be sufficient to consider $f(t, x)$ defined and continuous on a product set $J \times \Omega_{X}$, where $\Omega_{X}$ is any open set in $X$ chosen so that the statement of Theorem 4.1 becomes meaningful.) For convenience, it will be assumed that $X$ is a vector space over the complex number field but, with suitable interpretations, the result and its proof are valid if $X$ is a vector space over the reals.

It will be assumed that $C$ is in a Jordan normal form $C=\operatorname{diag}$ $(J(1), \cdots, J(\mu))$, where $J(j)$ is an $h(j)$ by $h(j)$ Jordan block with the eigenvalue $\lambda(j)$ on its diagonal and, if $h(j)>1,1$ on the subdiagonal. Correspondingly, write $x=\left(x_{1}, \cdots, x_{\mu}\right)$, where $x_{j}=\left(x_{j}^{1}, \cdots, x_{j}^{h(j)}\right)$ is an $h(j)$-tuple. With a similar renumbering of the components of $f(t, x)=$ $\left(f_{1}, \cdots, f_{\mu}\right)$, (4.6) becomes

$$
x_{j}^{1^{\prime}}=\lambda(j) x_{j}^{1}+f_{j}^{1}, x_{j}^{k^{\prime}}=\lambda(j) x_{j}^{k}+x_{j}^{k-1}+f_{j}^{k} \text { for } k=2, \cdots, h(j),
$$

for $j=1, \cdots, \mu$ or

$$
x_{j}^{\prime}=J(j) x_{i}+f_{j}(t, x) \text { for } j=1, \cdots, \mu .
$$

Let $\alpha$ denote one of the numbers $\operatorname{Re} \lambda(1), \cdots, \operatorname{Re} \lambda(\mu)$ and let an index $j, 1 \leqq j \leqq \mu$, be denoted by $p, q$, or $r$ according as $\operatorname{Re} \lambda(j)<$, $=$, or $>\alpha$. The set of indices $p$ and/or $r$ can be vacuous. Put

$$
h_{*}=\max _{q} h(q) \text {. }
$$

The linear system $x^{\prime}=C x$ has solutions of the form $x_{q}=e^{\lambda(q) t} P_{q}(t)$, $x_{j}=0$ for $j \neq q$, where $P_{q}(t)$ is an $h(q)$-tuple each component of which 
is a certain polynomial. The question to be discussed is when does. (4.6) have a solution which approximates this solution of $x^{\prime}=C x$ in a certain sense. Because of the fact that certain remainder terms may become larger than certain "principal" terms, the question will be formulated more precisely as follows:

Let $j_{0}$ be an integer and $\beta$ an arbitrary number subject to

$$
0 \leqq j_{0} \leqq h_{*}-1 \text { and } \beta+j_{0} \geqq h_{*}
$$

(so that $\beta \geqq 1$ ). Let $k=l(q), k(q)$ be the least and greatest values. of $k$, if any, satisfying

$$
1 \leqq l(q) \leqq k(q) \leqq \min (h(q), \beta) \text { and } h(q)-l(q) \leqq j_{0} .
$$

Let $c_{q}^{i}, l(q) \leqq i \leqq k(q)$, be numbers. Solutions $x(t)$ of (4.6) are sought. with the asymptotic behavior

$$
\begin{aligned}
& x_{q}^{k}=e^{\lambda(q) t}\left\{\Sigma_{I} t^{k-i} y_{q}^{i} /(k-1) !+t^{k-\beta} \Sigma_{I I} y_{q}^{i} /(k-i) !\right\}, \\
& x_{j}=e^{\alpha t} t^{1-\beta} y_{j} \text { if } \quad j \neq q,
\end{aligned}
$$

where

$$
y_{q}^{i} \rightarrow c_{q}^{i} \text { for } l(q) \leqq i \leqq k(q), y_{j}^{i} \rightarrow 0 \text { otherwise , }
$$

as $t \rightarrow \infty$. In (4.12), $\Sigma_{I}$ is the sum over the indices $i$ satisfying. $l(q) \leqq i \leqq \min (k(q), k)$ and $\Sigma_{I I}$ is the sum over the other indices $i$ on range $1 \leqq i \leqq k$.

Actually, unless estimates are furnished for $y_{q}^{i}(t)-c_{q}^{i}=o(1)$ as $t \rightarrow \infty$, the asympotic formulae (4.12) - (4.13) do not distinguish clearly between "principal terms" and "remainder terms." A more desirable. conclusion is obtained by replacing (4.13) by

(4.14) $y_{q}^{i}-c_{q}^{i}=o\left(t^{i-\beta}\right)$ for $l(q) \leqq i \leqq k(q)$ and $y_{j}^{i}=o(1)$ otherwise .

In this case, (4.12) and (4.12) imply

$$
\begin{aligned}
& x_{q}^{k}=e^{\lambda(q) t}\left\{\Sigma_{I} c_{q}^{i} t^{k-i} /(k-i) !+o\left(t^{k-\beta}\right)\right\} \quad \text { for } k=1, \cdots, h(q), \\
& x_{j}=o\left(e^{\alpha t} t^{1-\beta}\right) \text { for } j \neq q .
\end{aligned}
$$

Since $i \leqq k(q) \leqq \beta$ in $\Sigma_{I}$, it is seen that the principal terms are given by $\Sigma_{I}$. Roughly, the number of principal terms increase and the remainder terms become smaller as $\beta$ and/or $j_{0}$ increases.

Let (4.1) be identified with (4.12). This is essentially the change of variables introduced in [4]. For computational purposes, note that the map $x=R(t) y$ in (4.12) can be factored as follows: $x=Q(t) D(t) y$, where $x=Q(t) w$ is given by

$$
x_{j}=e^{J(j) t} w_{j} \quad \text { if } j=q, \quad x_{j}=e^{\alpha t} w_{i} \quad \text { if } j \neq q
$$


and $w=D(t) y$ is the (diagonal) map

$$
\begin{aligned}
& w_{q}^{k}=y_{q}^{k} \quad \text { if } \quad l(q) \leqq k \leqq k(q), \\
& w_{q}^{k}=t^{k-\beta} y_{q}^{k} \text { if } k<l(q) \text { or } k>k(q), \\
& w_{j}=t^{1-\beta} y_{j} \text { if } j \neq q .
\end{aligned}
$$

It is then readily seen that $R(t)$ is nonsingular and that the inverse map $y=R^{-1}(t) x$ is given by

$$
\begin{aligned}
& y_{q}^{k}=e^{-\lambda(q) t} \sum_{i=1}^{k}(-1)^{i} t^{k-1} x_{q}^{i} /(k-1) ! \text { if } l(q) \leqq k \leqq(q), \\
& y_{q}^{k}=e^{-\lambda(q) t} \sum_{i=1}^{k}(-1)^{i} t^{\beta-i} x_{q}^{i} /(k-i) ! \text { if } k<l(q) \text { or } k>k(q), \\
& y_{j}=e^{-\alpha t} t^{\beta-1} x_{j} \text { if } j \neq q .
\end{aligned}
$$

From (4.12), it follows that, for large $t$, the norm of $R(t)$ is majorized and minorized by a constant multiple of $e^{\alpha t} t^{\gamma}$, where $\gamma=$ $\max \left(h_{*}-\beta, h(q)-l(q)\right)$ and the max refers to $q$. Thus (4.10) shows that $\|R(t)\| e^{-a t} t^{-j_{0}}$ is bounded from above and below by positive constants as $t \rightarrow \infty$. Similarly, $\left\|R^{-1}(t)\right\| e^{\alpha t} t^{1-\beta}$ remains between two positive constants. Thus, there are positive constants $N, N^{\prime}, N_{1}, N_{1}^{\prime}$ such that for large $t$

$$
N^{\prime} \leqq\|R(t)\| e^{-\alpha t} t^{-j_{0}} \leqq N, \quad N_{1}^{\prime} \leqq\left\|R^{-1}(t)\right\| e^{\alpha t} t^{1-\beta} \leqq N_{1} .
$$

Since $e^{J(j) t}$ is a fundamental matrix for $x_{j}^{\prime}=J(j) x_{j}$, the factorization $R=Q D$ given above makes it easy to calculate the differential equation for $y$. It is of the form

$$
y^{\prime}=C_{0}(t) y+R^{-1}(t) f(t, R(t) y),
$$

where the linear part $y^{\prime}=C_{0}(t) y$ is

$$
\begin{aligned}
& y_{q}^{k \prime}=0 \text { if } l(q) \leqq k \leqq k(q), \\
& y_{q}^{k \prime}=(\beta-k) t^{-1} y_{q}^{k} \text { if } k<l(q) \text { or } k>k(q), \\
& y_{j}^{\prime}=\left[J(j)-\alpha I_{h}+(\beta-1) t^{-1} I_{h}\right] y_{j} \text { if } j=q,
\end{aligned}
$$

and $I_{h}$ is the $h(j)$ by $h(j)$ unit matrix.

Let $c=\left(c_{1}, \cdots, c_{\mu}\right) \in X$ be the vector where $c_{q}^{k}, l(q) \leqq k \leqq k(q)$, are the given constants and $c_{j}^{k}=0$ otherwise. The change of variables (4.3) reduces $(4.20)$ to

$$
z^{\prime}=C_{0}(t) z+R^{-1}(t) f(t, R(t) z+R(t) c),
$$

since $C_{0}(t) c=0$. Recall that $x=R(t) y$ is given by (4.12), and so $v=R(t) c$ is the vector $v=\left(v_{1}, \cdots, v_{\mu}\right)$ with components

$$
v_{q}^{k}=e^{\lambda(q) t} \sum_{I} t^{k-i} c_{q}^{i} /(k-i) ! \text { and } \quad v_{j}=0 \text { if } j \neq q \text {. }
$$


It is readily verified that $\left(L^{1}(X), L_{0}^{\infty}(X)\right)$ is admissible for $C_{0}(t)$, if these spaces refer to $t \geqq 1$, rather than $t \geqq 0$. In order to check this, it is sufficient to consider the three lines of (4.21) separately. The desired conclusion for the first two lines is obvious. In order to deal with the last line, consider

$$
y_{j}^{\prime}=\left[J(j)-\alpha I_{h}+(\beta-1) t^{-1} I_{h}\right] y_{j}+g_{j}(t),
$$

where $g_{j}(t) \in L^{1}$ for $1 \leqq t \leqq \infty$. A fundamental matrix for the homogeneous part of (4.24) is $U_{j}(t)=t^{\beta-1} e^{\left[J(j)-\alpha I_{h}\right]}$; cf. the last parts of (4.16), (4.17). There are positive constants $N_{0}, \gamma$ such that

$$
\left\|U_{j}(t) U_{j}^{-1}(s)\right\| \leqq N_{0} e^{-\gamma(t-s)} \quad \text { for } t \geqq s \geqq 1 \quad \text { if } \operatorname{Re} \lambda(j)<\alpha
$$

and

$$
\left\|U_{j}(t) U_{j}^{-1}(s)\right\| \leqq N_{0} e^{\gamma(t-s)} \quad \text { for } \quad 1 \leqq t \leqq s \quad \text { if } \quad R e \lambda(j)>\alpha .
$$

Thus, according as $\operatorname{Re} \lambda(j)<\alpha$ or $\operatorname{Re} \lambda(j)>\alpha$, (4.24) has the solution

$$
y_{j}(t)=\int_{1}^{t} U_{j}(t) U_{j}^{-1}(s) g_{j}(s) d s \quad \text { or } \quad y_{j}(t)=-\int_{t}^{\infty} U_{j}(t) U_{j}^{-1}(s) g_{j}(s) d s
$$

which tends to 0 as $t \rightarrow \infty$; cf. (2.1).

Theorem 2.1 will be shown to imply the following:

THeOREM 4.1. In the system (4.6), let $C=\operatorname{diag}(J(1), \cdots, J(\mu))$, where $J(j)$ is an $h(j)$ by $h(j)$ Jordan block with $\lambda(j)$ on its diagonal. Let $\alpha=\operatorname{Re} \lambda(j)$ for some $j$ and let $j$ be denoted by $p, q, r$ according as $\operatorname{Re} \lambda(j)<,=$, or $>\alpha$ and define $h_{*}$ by (4.9). Let $j_{0}$ be an integer and $\beta$ a number satisfying (4.10). Let $l(q), k(q)$ be the least and greatest integers, if any, satisfying (4.11). Let $f(t, x)$ be continuous for $t \geqq 0$ and all $x$. Let $c \in X$ be a constant vector with components $c_{j}^{k}=0$ if $j=p, r$ or $j=q$ and $k<l(q)$ or $k>k(q)$. Let there exist a number $\rho>0$ and a function $\lambda(t), 1 \leqq t<\infty$, such that

$$
\begin{gathered}
\int^{\infty} \lambda(t) d t<\infty \text { and }\left\|R^{-1}(t)\right\| \cdot\|f(t, R(t) z+R(t) c)\| \leqq \lambda(t) \\
\text { for }\|z\| \leqq \rho,
\end{gathered}
$$

where $x=R(t) y$ is given by (4.12). Finally, let

$$
m=\Sigma h(p)+\Sigma(h(q)-k(q)) .
$$

Then there exists an $m$ parameter family of solutions $x(t)$ of (4.6) satisfying the asymptotic formula (4.15).

The conclusion of this theorem can be stated in a different form: if $T>0$ is sufficiently large and the $m$ numbers $\left|y_{p 0}^{k}\right|$ and $\left|y_{q 0}^{k}\right|$, 
$k>k(q)$, are sufficiently small, then there exists a solution $x(t)$ of (4.6) for $t \geqq T$ satisfying the asymptotic formula (4.15) and the partial set of initial conditions $y_{j}^{k}(T)=y_{j 0}^{k}$ if $j=p$ and $j=q, k>k(q)$ in the $y$-coordinate system defined by (4.18) for $t=T$.

In view of (4.19), the last part of (4.25) is the same as

$$
e^{-\alpha t} t^{\beta-1}\|f(t, R(t) z+R(t) c)\| \leqq \lambda(t) \text { for }\|z\| \leqq \rho .
$$

The proof of Theorem 4.1 will show that this condition can be relaxed to the following pair of conditions

$$
\begin{gathered}
\left\|R^{-1}(t) f(t, R(t) z+R(t) c)\right\| \leqq \lambda(t) \text { for }\|z\| \leqq \rho, \\
e^{-\alpha t} t^{\beta-k}\left|f_{q}^{k}(t, R(t) z+R(t) c)\right| \leqq \lambda(t) \text { for }\|z\| \leqq \rho, 1 \leqq k \leqq k(q) .
\end{gathered}
$$

Note that if $f(t, x)$ has a majorant of the form

$$
\|f(t, x)\| \leqq \psi(t)\|x\|,
$$

then (4.19) shows that (4.25) holds for all choices of the constants $c_{q}^{k}, l(q) \leqq k \leqq k(q)$, if

$$
\int^{\infty} t^{\beta+j_{0}-1} \psi(t) d t<\infty .
$$

When (4.29) is replaced by

$$
\|f(t, x)\| \leqq \psi(t) \varphi(\|x\|),
$$

where $\varphi(\sigma)$ is a nondecreasing function of $\sigma, \sigma>0$, then a sufficient condition for (4.25) is that

$$
\int^{8}\left\|R^{-1}(t)\right\| \psi(t) \varphi((\rho+\|c\|)\|R(t)\|) d t<\infty
$$

for some $\rho>0$. If, in addition, $\psi(t)$ is a nonincreasing function, (4.32) holds if $\alpha>0$,

$$
\int^{\infty} \sigma^{-2}|\log \sigma|^{\beta+j_{0}-1} \psi(a \log \sigma) \varphi(b \sigma) d \sigma<\infty
$$

for some $a<1 / \alpha$ and $b>N\|c\|$; or if $\alpha<0$,

$$
\int_{+0} \sigma^{-2}|\log \sigma| \psi(a|\log \sigma|) \varphi(b \sigma) d \sigma<\infty
$$

for some $a<-1 / \alpha$ and $b>N\|c\|$; or if $\alpha=0$,

$$
\int^{\infty} \sigma^{-1+\beta / j_{0}} \psi\left(\sigma^{1 / j_{0}}\right) \varphi(b \sigma) d \sigma<\infty
$$

for some $b>N\|c\|$. 
If $\varphi(\sigma)=1$, then (4.31) becomes

$$
\|f(t, x)\| \leqq \psi(t)
$$

and (4.32) is implied by

$$
\int^{\infty} e^{-\alpha t} t^{\beta-1} \psi(t) d t<\infty .
$$

The inequality (4.36) is, of course, required only for $(t, x)$ of the form $(t, x)=(t, R(t) z+R(t) c),\|z\| \leqq \rho$ for some $\rho$ and $t \geqq 1$. This set of $(t, x)$ is contained, for example, in

$$
\left\{(t, x): t \geqq 1 \text { and }\|x\| \leqq N e^{\alpha t} t^{j_{0}}(\rho+\|c\|)\right\} .
$$

Proof of Theorem 4.1. The considerations preceding the statement of Theorem 4.1 show that if the assumption in the last part of (4.25) is relaxed to (4.27) and if (4.15) in the conclusion is relaxed to (4.12)-(4.13), then the corresponding result is a consequence of Theorem 2.1.

It has to be shown that if $x(t)$ is a solution of (4.6) for large $t$ satisfying (4.12)-(4.13), then (4.14) also holds. It is seen from (4.20) and the first part of (4.21) that, for $l(q) \leqq k \leqq k(q)$,

$$
y_{q}^{k}(t)=c_{q}^{k}-\int_{t}^{\infty}\left[R^{-1}(s) f(s, R(s) y(s))\right]_{q}^{k} d s,
$$

where $\left[R^{-1} f\right]_{q}^{k}$ indicates the $(q k)$ th component of $R^{-1} f$. Since $y=R^{-1} x$ is given by (4.8),

$$
y_{q}^{k}(t)=c_{q}^{k}-\int_{t}^{\infty} e^{-\lambda(q) s} \sum_{i=1}^{k}(-1)^{i} s^{k-1} f_{q}^{i} d s /(k-i) !,
$$

where the argument of $f_{q}^{k}$ is $(s, R(s) y(s))$. By (4.28)

$$
\left|y_{q}^{k}(t)-c_{q}^{k}\right| \leqq e \int_{t}^{\infty} s^{k-\beta} \lambda(s) d s \leqq e t^{k-\beta} \int_{t}^{\infty} \lambda(s) d s
$$

for large $t$ since $y(s) \rightarrow c$ as $s \rightarrow \infty$. This gives the first part of (4.14) and completes the proof of the theorem.

It can be remarked that one of the keys to Theorem 4.1 is the change of variables $x=R(t) y$ of (4.12) from [4]. Corduneanu's theorem is only one of many ways of dealing with the resulting equation (4.20).

6. Addendum. The object of this section is to make a remark about Corduneanu's Theorem I in [1]. In this theorem, $X$ is permitted to be a Banach space of finite or infinite dimension. In case $\operatorname{dim} X=\infty$, it is assumed that $X_{0 \mathscr{g}}$ is closed and has a closed com- 
plement $X_{1 \mathscr{D}}$ in $X$. It turns out that one can establish an analogous existence theorem without the assumption that $X_{0 \mathscr{D}}$ is closed or has a closed complement (but specification of a condition of the type $P_{0 \mathscr{D}} x(0)=\xi_{0}$ and corresponding uniqueness is lost).

The notations above will be used. Here, "locally integrable" means "locally Bochner integrable" and $\Sigma$ again denotes the ball $\left\{x(t): x(t) \in \mathscr{D},|x|_{D} \leqq \rho\right\}$ in $D, \rho>0$.

Theorem 6.1. Let $X$ be a Banach space $(\operatorname{dim} X \leqq \infty)$ and $A(t)$ locally integrable on $J$. Let $A(t), f(t, x)$ satisfy (a) $(\mathscr{B}, \mathscr{D})$ is admissible for $A(t)$ and (b) $x(t) \rightarrow f(t, x(t))$ is a continuous operator from $\Sigma \subset \mathscr{D}$ to $\mathscr{B}$ satisfying

$$
\left|f\left(t, x_{1}(t)\right)-f\left(t, x_{2}(t)\right)\right|_{\mathscr{D}} \leqq \theta\left|x_{1}-x_{2}\right|_{\mathscr{D}} \text {, where } 0<\theta<1,
$$

for all $x_{1}(t), x_{2}(t) \in \Sigma$ and some constant $\theta$. Let $m=|f(t, 0)|_{\mathscr{B}}$. Then there exists a constant $K$, depending only on $A(t), \mathscr{B}$ and $\mathscr{D}$, such that if

$$
K m /(1-\theta) \leqq \rho,
$$

then (1.3) has at least one $\mathscr{D}$-solution $x(t) \in \Sigma$.

The proof will depend on a procedure due to L. M. Graves [Duke Mathematical Journal, vol. 17 (1950), pp. 114-111] and results of Massera and Schäffer [7].

Proof. By Theorem 2.2 in [7], condition (a) implies that there exists a constant $K$, depending only on $A(t), \mathscr{B}$ and $\mathscr{D}$, such that if $g(t) \in \mathscr{B}$, then (1.2) has at least one $\mathscr{D}$-solution $x(t)$ satisfying

$$
|x|_{\mathscr{D}} \leqq K|g|_{\mathscr{O}} \text {. }
$$

In order to find a solution for (1.3), form a sequence of successive approximations $x_{0}(t), x_{1}(t), \cdots$ as follows: Let $x_{0}(t) \equiv 0$. Let $x_{1}(t)$ be a $\mathscr{D}$-solution of (1.2), when $\mathrm{g}(t)=f(t, 0) \in \mathscr{B}$, satisfying

$$
\left|x_{1}\right|_{\mathscr{D}} \leqq K|f(t, 0)|_{\mathscr{B}}=K m \text {. }
$$

Note that $K m<\rho$, so that $x_{1}(t) \in \Sigma$. If $x_{0}(t), \cdots, x_{n-1}(t)$, where $n \geqq 2$, have been defined and are elements of $\Sigma$, define $x_{n}(t)$ by letting $x_{n}(t)-x_{n-1}(t)$ be a solution of (1.2), with

$$
g(t)=f\left(t, x_{n-1}(t)\right)-f\left(t, x_{n-2}(t)\right),
$$

satisfying (6.3). Thus, by (6.1),

$$
\left|x_{n}-x_{n-1}\right|_{\mathscr{D}} \leqq K \theta\left|x_{n-1}-x_{n-2}\right|_{\mathscr{D}} \text { for } n \geqq 2 \text {. }
$$


Hence, by induction,

$$
\left|x_{n}-x_{n-1}\right|_{\mathscr{D}} \leqq K \theta^{n-1} m \text { for } n \geqq 1 \text {. }
$$

Note that $x_{n}=x_{1}+\left(x_{2}-x_{1}\right)+\cdots+\left(x_{n}-x_{n-1}\right)$ has a norm satisfying

$$
\left|x_{n}\right|_{\mathscr{D}} \leqq K m \sum_{j=0}^{n-1} \theta^{j}<K m /(1-\theta) \leqq \rho .
$$

Thus $x_{n}(t) \in \Sigma$ and so $x_{n}(t)$ can be defined for all $n=1,2, \cdots$.

Since $x_{1}(t)$ satisfies

$$
x_{1}^{\prime}=A(t) x_{1}+f\left(t, x_{0}\right),
$$

and $x_{n}(t)-x_{n-1}(t)$ satisfies

$$
\left(x_{n}-x_{n-1}\right)^{\prime}=A(t)\left(x_{n}-x_{n-1}\right)+f\left(t, x_{n-1}\right)-f\left(t, x_{n-2}\right)
$$

for $n \geqq 2$, it is clear that

$$
x_{n}^{\prime}=A(t) x_{n}+f\left(t, x_{n-1}\right) \text {. }
$$

Furthermore,

$$
x(t)=\lim _{n \rightarrow \infty} x_{n}(t)=x_{1}+\sum_{j=2}^{\infty}\left(x_{j}-x_{j-1}\right)
$$

exists in $\mathscr{D}$ and is an element of $\Sigma$. Since (b) implies that $f\left(t, x_{n-1}\right)$ $\rightarrow f(t, x(t))$ in $\mathscr{B}$ as $n \rightarrow \infty$, it follows from Lemma 2.1 in [7] that $x(t)$ is a $\mathscr{D}$-solution of (1.3). This proves Theorem 6.1.

\section{REFERENCES}

1. C. Corduneanu, Sur certaines systèmes différentielles non-lenéaires, Analele Știinițifice ale Universității “Al. I. Cuza” din Iași, Sect. I, 6 (1960), 257-260.

2. O. Dunkel, Regular points of a system of homogeneous linear differential equations of the first order, Proc. Amer. Acad. Arts and Sci., 38 (1902-3), 341-370.

3. J. Hale and N. Onuchic, On the asympotic behavior of solutions of a class of differential equations, Contributions to Differential Equations, to appear.

4. P. Hartman and A. Wintner, Asymptotic integrations of linear differential equations, Amer. J. Math., 77 (1955), 45-86.

5. P. Hartman and A. Wintner, Asymptotic integrations of non-linear differential equations, Amer. J. Math., 77 (1955), 692-724.

6. J. L. Massera, Sur l'existence de solutions bornées et périodiques des systèmes quasilinéaires d'équations différentielles, Annali di Matematica Pura ed Applicata, ser. IV, 51 (1960), 95-105.

7. J. L. Massera and J. J. Schäffer, Linear differential equations and functional analysis. IV, Mathematische Annalen, 139 (1960), 287-342.

8. C. Olech, On the asymptotic behavior of the solutions of a system of ordinary nonlinear differential equations, Bulletin de l'Académie Polonaise des Sciences, cl. III, 4 (1956), 555-561.

9. J. J. Schäffer, Linear differential equations and functional analysis. VI, Mathematische Annalen, 154 (1962), 354-400.

THE JOHNS HopKINS UNIVERSITY

Rias and Faculdade de Filosofia, Ciencias E letras, Rio Claro, S.P., Brazil 
